\title{
A Deep Unity between Scientific Disciplines
}

\author{
Cédric Gaucherel ${ }^{1,2}$ \\ ${ }^{1}$ French Institute of Pondicherry, IFP-CNRS, Pondicherry, India \\ ${ }^{2}$ UMR AMAP-INRA, Montpellier, France \\ Email: cedric.gaucherel@ifpindia.org
}

Received March 21 $1^{\text {st }}$,2013; revised April 21 ${ }^{\text {st }}$, 2013; accepted May $1^{\text {st }}, 2013$

\begin{abstract}
Copyright (c) 2013 Cédric Gaucherel. This is an open access article distributed under the Creative Commons Attribution License, which permits unrestricted use, distribution, and reproduction in any medium, provided the original work is properly cited.
\end{abstract}

\begin{abstract}
Are scientific disciplines really different? This question often crystallizes into the old debate: Are Physics and Biology different? If Physics and Biology worked on highly different entities (objects), or if they had highly different methods, it would be straightforward to close the debate by a negative answer. However, if we cannot identify any differences, we should explore more deeply the status of the laws found in Physics and questioned in Biology. By slightly modifying the definition of what is a law, I argue here that both disciplines possess some laws exhibiting various "degrees of confirmation". I finally propose explanations for why $\mathrm{P}$ and $\mathrm{B}$ give the illusion differing radically, although they both belong to the same continuum of a unified scientific domain.
\end{abstract}

Keywords: Scientific Law; Unicity; Contingency; Universality; Neutral Model; Evolution; Gravitation.

\section{Introduction}

The old debate on whether Physics (P) and Biology (B) belong to the same culture has recently been reactivated. Here by culture, we are not thinking to the social part of the scientific activity, rather than to the deep nature of their studied objects. Some scientists claim that physicists seek simplicity in universal laws, while biologists revel in complex interdependencies and specific processes (Keller, 2007). Still others admit these differences in method, but claim that they hold for both physical and biological studies (Harte, 2002). In this paper, I argue that $\mathrm{P}$ and $\mathrm{B}$ do not differ, that, in particular, physical and biological entities are not different in nature, and that physical and biological disciplines use a similar method (Pombo et al., 2012). $\mathrm{P}$ and $\mathrm{B}$ both build some laws to interpret their observations. The central argument of this paper proposes a slightly modified definition of what is a law to accommodate their possible differences. I finally propose an explanation of why P and B often give the illusion differing radically between themselves.

There is no doubt that $\mathrm{P}$ searches for laws, a law being viewed in the broad sense as a statement expressing an observed regularity. I will not debate the relevance of laws (Cartwright, 1983), but instead treat them as useful models of reality to explore possible differences between $\mathrm{P}$ and $\mathrm{B}$. According to Carnap, in particular, a scientific law is said to be "universal" if it is a principle that is observed to operate at all times and all places, without exception (Carnap, 1974). Particle collisions conserve the total energy of the system. This is always observed, and there are no exceptions to it. It is a universal law. It is less clear that B possesses such universal laws.

When subjected to careful scrutiny, it seems that $\mathrm{P}$ does not have universal laws either. Quantum theory indeed highlights processes that are intrinsically probabilistic, meaning thereby that we cannot formulate a deterministic statement based on observations. This limitation is not a result of our ignorance, as was shown by W. Heisenberg, but rather a result of the intrinsic structure of our world. This view suggested a need to define what has been called "statistical" laws, which assert that regularity occurs only in a certain percentage of cases instead of in all cases. Even with this less constraining definition, are we allowed to assert that $\mathrm{B}$ has any laws at all that are applicable? This is the main question addressed in this paper, the central idea being that any differences in (or in the use of) laws in B and $\mathrm{P}$ would definitively discriminate these two disciplines.

For this reason, this paper proceeds in three successive stages. In the first part, it examines, with examples, whether physical and biological entities are similar in nature. Living systems are often perceived as unique, contingent (i.e. dependent) on events, and ultimately irreversible entities. Such a view can lead to a feeling that the functioning of living systems is less universal (i.e. less systematic) than is that of inert systems. If true, either of these properties would be sufficient to attest to a radical difference between P and B. Another way P and B might differ relates to the specific methods they use. One of the most commonly used scientific methods today is the hypothetico-deductive model, formulating a hypothesis and validating (or invaliddating) it on the basis of experiments. Practically, physicists and other scientists often use "neutral models" to verify whether their models (intentionally forgetting the process being studied) correctly represent observations or not. The steadily growing use of neutral models in B will help us to study hypothetico-deductive methods common to $\mathrm{P}$ and $\mathrm{B}$. The type of method used in each discipline, and the related considerations, make up the substance of the second part.

In the third and last stage, I will discuss the two previous arguments (namely, whether physical and biological entities and methods are similar) in order to better identify the remaining specificities of $\mathrm{P}$ and $\mathrm{B}$. In particular, it will be necessary here to explore the perceptions we have of $\mathrm{P}$ and $\mathrm{B}$ processes. The 
arguments presented in the previous stage highlight the crucial difference between the probability of a process to occur, and the probability of its associated law to exist. Following Carnap (1974), I suggest here a resort to the concept of a "probabilistic" law, rather than a "universal" law, to analyze the "degree of confirmation" we have from the laws being applied. Such a probabilistic view of $\mathrm{P}$ and $\mathrm{B}$ suggests moving the qualitative differences often be perceived between the two disciplines into the quantitative continuum of a unique scientific domain. Moreover, this conceptual continuum may be explained and justified by the remaining differences in nature as discussed above.

\section{Are $P$ and $B$ Different in Their Nature?}

At first sight, the inert entities and the living entities studied by $\mathrm{P}$ and $\mathrm{B}$ respectively seem to differ highly in their nature. If true, this assertion would support acceptance of different scientific disciplines for their respective studies. Yet, what do we mean when we talk about the nature of entities? $\mathrm{P}$ and $\mathrm{B}$ are wide domains of research, studying entities as varied as minerals or stones on the one hand, and cells or individuals on the other hand. I will not address the Holism-Reductionism debate here, which is out of the scope of this paper and would bring a sterile view of P and B differences (Trepl \& Voigt, 2011). We need to list some of the most important properties defining these entities and their natures in order to examine whether they are found to be similar in $\mathrm{P}$ and $\mathrm{B}$.

One of the most important properties of B objects that has been cited in the past is their unicity (Pesic, 2002). A cell or an individual always exhibits sharp differences from its neighbor, although a star or a particle is supposedly similar to its neighbor. I will show in the next sub-section that this somewhat simplistic view of unicity is not a systematic rule. A second important property that apparently differs between physical entities and biological entities is the contingency involved in their construction (Gould, 1989). Contingency is the fact that we observe an entity as it is, mainly (or only) because of a succession of events that may not have occurred. We often have the feeling, although not yet demonstrated, that most living entities are the result of a combination of singular events. These events, and no other events, have occurred. I will show that this is true in some cases, and false in others.

Furthermore, unicity and contingency may be two facets of the same important property. An object that is contingent, such as the brain of an individual, is also unique, in that it would certainly be necessary to replicate rigorously the same sequence of events as must have been followed in the original if one were to wish to re-construct the same brain. Could we imagine different events sometimes leading to exactly the same entity? On the other hand, it is highly probable for a unique entity to be the result of contingent events, as the events of its construction would inevitably lead to the same entity. These events, if it should ever be possible to make them occur again, would lead to an entity not unique any more. So, unicity and contingency are not identical concepts, but they remain closely linked due to a third hidden property: irreversibility. Indeed, specific events are determinant in some entity construction, especially in case of irreversible events (Nicolis \& Prigogine, 1977). In the case of reversible events, the entity depending on them can systematically come back to its previous state, and is therefore no more either contingent or unique.

\section{$B$ and P: Unicity}

First, the fact that Life is unique (we know only one single sample of it!) suggested to some biologists to highlight the specificity of $B$, and to reject the Popperian view of scientific research in evolutionary B (Mayr, 2004). Indeed, if we knew of a second sample of Life, we would start to be able to refute some hypotheses based on the Life we know. In astrophysics, scientists similarly handle a unique entity: the Universe. This remark is true at finer scales too, as Earth (or at least the geosphere component of it) is a unique entity studied by geophysicists.

Yet, we all know that these assertions are frequently debated. For example, it has been argued that each trait convergence (similar traits observed between different species) is another confirmation that Evolution acts in a reproducible manner in similar conditions (Morris, 2010). While we are on the way to discovering planets similar to Earth, we are not sure how similar they will be. Furthermore, there are active discussions on about multiverses, challenging the usual limited definition of the universe (Tegmark, 2003). On the other hand, the unicity that may be true for evolutionary B is basically false in molecular or cell B. There exist as many molecules and as many cells as necessary to test biological hypotheses. This observation is very similar to the one that there exist plenty of rigorously similar particles and nuclear entities to be studied in high-energy P.

Regardless of what elements might be true, and what might not, what is important for our discussion are the similarities between physical and biological entities. For the sake of the discussion, I question here whether this dichotomy between unicity and multiplicity of entities is partly related to scale or not. At scales larger than our human scale (e.g. meters to kilometers and seconds to years), Life and the Universe appear unique. At least, this seems to be the case to our knowledge, and our knowledge is strongly limited at higher scales. Entities at lower scales can be found in many samples, down to cells and particles. At intermediate human scales, geophysics and ecology offer interesting similarities too. They possess an intermediate status ranging from unique Earth geosphere and biosphere (Lovelock, 2003), through a few plates and continents, down to a multiplicity of geophysical systems and ecosystems.

Finally, entities with inert properties and those with living properties appear to exhibit very similar natures, both covering the whole range of diversity, from a unique entity to high numbers of replicates (of events). Therefore, it seems that the commonly observed acceptance of unicity of physical and biological entities is more closely linked to their associated scales than to their domains.

\section{$B$ and $P$ Contingency}

Secondly, the life sciences often present contingency as a property responsible for the specificities of biological entities (Gould, 1989). Hence, we may observe living entities as they are only due to a combination of isolated and improbable events (sometimes called "accidents"). These events may be random or not. Most biological entities are supposed to be contingent. A cell or an individual is highly dependent upon events and environmental states leading to its construction. At the same time, many physical entities may be contingent too. Plate tectonics leads to a contingent geology on earth, precisely the one we observe today. Planetary astrophysics, studying how the so- 
lar system has formed, is also a clear example of contingent physical entities. These systems would not have been the same with a poorer composition of the surrounding environment or, on a longer time scale, with another date of the Universe's formation.

Here again, there is a debate about these possible contingent entities. For example, it has been recently suggested that evolutionary trait convergences are confirmations that Evolution acts in a similar manner when exposed to similar conditions (Morris, 2010). The recent discoveries of extrasolar planets in distant planetary systems also highlight the possibility that a different set of astrophysical events may lead to approximately similar entities, thus being less and less contingent (Beaulieu et al., 2006).

Whatever the degree of contingency in physical and biological entities, the property appears to be present in these domains. Finally, P and B both study entities and events that have similar properties and similar requirements of universality. These entities may sometimes be unique, sometimes not; they may sometimes be contingent, sometimes not. Contingency and unicity could be two facets of the same property: systems symbolizing unicity, such as Life or the Universe, are clearly a result of a succession of highly improbable events. It must also be remembered that events exhibiting these properties are usually irreversible events. The next subsection explores this critical property of irreversibility to show up possible differences between $\mathrm{P}$ and $\mathrm{B}$.

\section{Irreversibility}

Most physical events appear to be reversible. In interactions among particles and those among stars, the equations governing their movements keep the same shape when time is reversed. Yet, our experience with everyday physical events tells us that these macroscale events are irreversible. The decay of a fruit and the mixing of milk in a cup of coffee are typical and familiar examples. It is even easier to find irreversible events in B, such as cell mitosis or the birth of an individual. Is such similarity between P and B real or only apparent? And in the case of an apparent similarity, why is there a difference?

Irreversibility is not a property of time: it is rather a property of the objects and the events studied. A wide range of physical processes have been discovered to be irreversible, since Newton's pioneering work on viscosity. We might also mention Coulomb's law, the Navier-Stokes equations, the laws of thermodynamics, Fick's laws of diffusion, the Joule effect, etc. Scientists waited for the thermodynamic synthesis, in the early 19th century, by S. Carnot and R. Clausius, to understand this observation as a natural tendency for closed systems to go towards disorder (i.e. the second law of thermodynamics). So, although many physical processes appear to be reversible, ireversible processes at macroscale are common too. More recently, in the second half of the 20th century, physical ireversible processes have also been observed at microscale, in particle physics experiments on matter's symmetry. Time's symmetry, as also the parity and charge symmetries, have been violated in the now famous CP (charge-parity) violation experiment by J. Cronin and V. Fitch in 1964.

So, are physical events reversible or not? L. Boltzmann already suggested a century ago how to explain this apparent paradox. Considering that a macroscale entity or event implies a huge number of constitutive entities, it would seem sensible to adopt a probabilistic view to understand the entities' dynamics. A reversible process is in theory always possible, whatever its scale. Yet, this large number of constitutive macroscale entities is the only reason why such a reversible change is highly improbable (Balian, 1991). One liter of water contains at least 1025 molecules, a far higher count than physics experiments yield for a comparable measure of particles. The probability for a system to go back to its previous state is infinitesimal in case of macroscale systems, varying inversely as the number of its components. In another context, H. Poincaré had quite graphically suggested that we might have to wait far longer than the age of the Universe for a chance to be able to observe such a reversible change at macroscale.

Finally, B processes often appear irreversible mainly due to their high number of interacting components. They possess a very low and even negligible probability to revert to their previous state (Nicolis \& Prigogine, 1977). In this context, each event plays an important role in the entity's construction and fate. Such entities are unique and are contingent. Some physical processes are irreversible too. For example, Earth's components are far more numerous than those encountered in reversible microscale experiments. Irreversibility is found in both P and B. This appears obvious as both domains study entities that have variously a high number or a low number of components, thus variously exhibiting unique, contingent and irreversible characteristics, whatever their scale.

\section{The Scale Issue}

Microscale events are reversible. At the same time, we have seen that irreversible events are frequently found at intermediate scales, e.g. at human scales. Hence, we may wonder in particular why stars, spanning very large spatial and temporal scales and with an enormous number of particle components, should again behave in a reversible way. Their growth and movement equations are indeed symmetrical with respect to time. To understand this, it is necessary to analyze the four fundamental interactions found in P. Strong and weak nuclear forces only concern very fine scales due to their very short interaction ranges (between 10 - $18 \mathrm{~m}$ and 10 - $15 \mathrm{~m}$ ). Despite this, they are much stronger than the gravity (1025 to 1038 times more) governing the movements of stars and galaxies. Gravity has an infinite range of influence. The electromagnetic force, also having an infinite range of influence, does not play a role at such large scales (planetary systems range in scale from $1012 \mathrm{~m}$ to $1014 \mathrm{~m}$ ). Unlike the gravitational force, the electromagnetic force can be absorbed, transformed, or deflected. Ratios of the measures of the strength of the forces to the sizes of the associated entities for gravitation are very similar to the corresponding ratios for nuclear forces (both strong and weak). A planetary system is approximately 1022 to 1026 times larger than an atomic system, while its associated fundamental forces are approximately 1025 to 1038 times stronger.

This scaling ratio partly explains why planetary systems behave similarly to atomic systems, although both act under different fundamental forces. The ranges of their interaction combined to their relative strengths are probably responsible for their similarity. The gravitation force has the effect of reducing the interactions among the large and massive entities under its influence by separating those entities in such a way that they behave as if they were new, coherent and independent or unrelated entities. In a sense, a star behaves under gravitation much 
as a particle would behave under nuclear forces. The electromagnetic force may have a similar effect, but as it can be deflected, it loses its influence at larger scales. Indeed, entities located between two other entities hide their respective influences, and indirectly reduce the force range. Hence, gravitation affects matter at large scales, and ceases to be perceptible at finer scales and on entities at such scales. This is not the case with entities found at intermediate scales, such as our close environment, which is governed by the fundamental force of electromagnetism and, in a reduced measure, by the other forces. I suggest that these differences could partly explain why the electromagnetic force increases entity interactions and favors irreversible events and entities.

Finally, it is worth observing that both $\mathrm{P}$ and $\mathrm{B}$ cover almost all scales. Hence, we can now assert that both exhibit in their own individual manner the complementary faces of the nature of entities.

\section{Are $\mathbf{P}$ and $B$ Different in Their Methods?}

If physical and biological entities are similar in nature, do their associated disciplines also work in similar ways? We need to scrutinize the specific methods or approaches of these domains before we are able to answer this question. Now, how do scientists usually go about achieving an understanding of an event, and providing a demonstration that there is a process that drives the functioning of physical and biological entities? When we talk of a process, we mean something that is happening on its own, by chance as it were. Although chance itself might be considered a process (called random), this process possesses a nature very different from that of non-random processes. Furthermore, the inherent stochasticity of our world adds varying amounts of noise to every process, thus blurring it. Only an uncovering of such a veil will allow us to see the process at work behind the noise.

A simple and easy-to-manage method for this purpose would be to formulate a hypothesis stating that the supposed process in question is present, and that it could be differentiated from a purely random process, under certain conditions. We would, of course, identify the conditions necessary to succeed in controlling (i.e. reducing) the inherent stochasticity of the process. We would know when such conditions occur, and when they do not occur, and we would know their nature. Such a method of searching for a hidden process is the familiar process of choosing a null hypothesis. A Neutral Model (NM) is a model that deliberately avoids a process of the studied entity or event, with the idea that it actually drives it (Nitecki \& Hoffman, 1987). When not invalidated, the NM suggests that chance may be a "process" sufficient to drive and to explain the observed entity. Conversely, when the NM is invalidated (i.e. rejected or falsified), it reinforces our belief that the process being studied is at work. Ultimately, the NM helps to define new alternative hypotheses, foundations of a new class of processes.

$\mathrm{P}$ has been using NM for a long time (Fisher, 1966). Chance being ubiquitous in physical processes, and because of the huge amounts of data provided by instruments and sensors in $\mathrm{P}$, it has become common, and meaningful, to use NM to test hypotheses. The recent appearance of powerful computers capable of analyzing the very large volumes of data generated has also contributed to enhancing the use of NM. As far back as thirty years ago, B's efforts to develop NM were documented (Nitecki \& Hoffman, 1987), and the progressive increase in collected data in the biological sciences, as well as the recognized influence of stochasticity, together present a strong argument in favor of NM use in this domain. These two factors may not explain, by themselves, the recent spread of NM in B. Still, that fact provides a strong clue, in my opinion, that $\mathrm{P}$ and B adopt a similar scientific method.

\section{Neutral Models in Biology}

The growing recourse to NM in the life sciences in recent decades is a clear departure from past practice. The variability of neutrality possible in a neutral model enables one to develop increasingly chance-driven models, and choose from among them the most parsimonious model, ensuring, of course, that it has not been invalidated. The importance given to chance becomes obvious in this approach. Compared to P, B today has many NM that have not yet been rejected, thus underlining the dominant role of chance in biological and ecological processes, as illustrated below.

The neutral-community theory proposes that species of the same community are not distributed according to their niche requirements (i.e. the habitat and ability to use resources), but according to random processes of dispersal limitation and game theory interactions (Hubbell, 2001). Species might all be ecologically equivalent in terms of migration and extinction processes. Although still debated, this hypothesis has not been completely rejected to date (Chase, 2005). Interestingly, specialists have started to believe that this view may be true at some scales (functional groups and biogeographic regions), but false at others (for example, at landscape scales). This anomaly may be due to habitat heterogeneities or to other spatial properties at intermediate scales.

Landscape-neutral models have been developed to simulate landscape structures and landscape functioning without explicitly using the processes and rules usually favored to generate these landscapes (Gardner et al., 1987). Landscape-neutral models attempt to generate natural as well as anthropogenic landscapes, and grid-based or vector-based landscapes (Gaucherel et al., 2006). But how far are observed landscapes from a neutrally-generated landscape? It seems today that almost-random statistical rules are often capable of building a high diversity of relatively realistic landscapes.

Correlated random walks appear to be the best strategy to use in searching for a particular resource when its distribution is unknown. In a similar spirit, indeed, several NM have been developed to improve our understanding of animal movements (i.e. trajectories). It has been proposed that most animal movements are equivalent to some weighted random walks called Lévy flights and Lévy walks (Viswanathan et al., 1999). This observation starts to be wrong when resources are aggregated in patches, suggesting that animals move more straightforwardly between resource patches and more erratically inside patches (Benhamou, 2007). Here again, we quantify with the help of this NM how far from random walks animals move. Such estimation between various NM appears to help in quantifying the role of stochasticity in these processes.

The neutral genetic theory proposes that Life experiences a genetic drift when some neutral genes (i.e. genes without phenotypic expression) are fixed by chance in a genome (Kimura, 1983). We know that evolution of Life is a complex combination of various forces among which some are neutral. Hence, these genes are not fixed by a natural selection process, but 


\section{GAUCHEREL}

rather by a neutral process. Stochasticity appears here to be systematically associated with evolutionary processes.

\section{Neutral Models: On the Way to Build a Law}

Finally, NM are not restricted to specific scales. NM in the life sciences range from broad scales to fine scales over a wide sweep of living entities, often crossing many scales simultaneously. Furthermore, self-similar NM, characterized by scaleinvariant (also called fractal) properties of the studied entity, are surprisingly ubiquitous in the life sciences. For example, the lungs, most tree and phytoplankton species and some vegetation or land cover patterns have been discovered to be selfsimilar (Scanlon et al., 2007; Gaucherel, 2011). Self-similar patterns highlight processes that are uniform across scales, over several magnitudes of orders. They are linear in a logarithmic plot, the so-called power laws, indicating that these patterns have no peculiar scale. Such perfect NM are always false, in a sense, as are all models, but they carry part of the real nature of the entities studied.

Which part of it? Self-similar NM capture a kind of system self-organization that is not yet understood. They tell almost nothing about the entities' functioning and are not able to explain processes generating the observed scaling pattern (Halley et al., 2004). Yet, many scientists think that ubiquitous selfsimilar patterns pave the way to a more universal self-organization principle of living entities (Kauffman, 1993). The selforganization principle hypothesizes that many processes may be interpreted through a kind of optimizing principle, sometimes called preferential attachment (D'Souza et al., 2007; Barabasi \& Albert, 1999).

This attempt too is a good example of what could one day become a law, or at least a principle, in B. NM are not laws but, when not rejected, they help us to understand by the neutral hypothesis the widest range of observations in a specific field. In this case, chance is sufficient to explain the observation. When NM are rejected, they help to define a new alternative hypothesis, new processes potentially serving as foundations of a natural law. Such a new hypothesis may progressively become a law, in the sense that every single observation in the discipline could then be interpreted in the context of this law. The following part of this paper intends to explore in more detail what a law is and which differences may exist between physical and biological natural laws. Finally, it appears that both $\mathrm{P}$ and $\mathrm{B}$ are largely using NM to test their hypotheses. This is a strong clue that they appear to have a similar way of working. They are very similar, and this similarity is becoming increasingly apparent in their methods and in relation to their construction of laws.

\section{Differences between B and P: Universality}

We have so far not identified in the course of our arguments any significant differences between $\mathrm{P}$ and $\mathrm{B}$, either in the nature of their entities, or in their scientific methods. Yet, we all have the feeling that $\mathrm{P}$ and $\mathrm{B}$ differ. For example, we have the intuittive impression that $\mathrm{P}$ encounters more "universal" processes and events than B does. Would there be a realistic basis for such an impression?

For the purpose of dealing with questions such as this, we need to have more accurate definitions and to determine whether they are appropriate or not. A process is an outcome of chance. When a (possibly unknown) process is recurrent and never fails, we usually call it a principle or a law. Newton's law of universal gravitation and the laws of thermodynamics are familiar examples of physical laws. Laws differ from theories in that they do not give any process and they do not formalize this process of their associated events. As laws are based on empirical constructions, i.e. observations, they are often found to be false when extrapolated. Newton's law of gravitation only applies in weak gravitational fields, and the second law of thermodynamics appears to be violated by random fluctuations of the system close to equilibrium (Evans et al., 1993). At the same time, I also have the feeling that theoretical entities are more valid than laws (Cartwright, 1983), as they persist over a longer span of time, but I still maintain that laws remain useful tools with which to understand the scientific representations of reality that we build.

Does this definition help us to understand what a universal law is? When all observations of a field are explained by a particular law, it may be called a "universal law" (Carnap, 1974). A universal law suffers no exception; never. Yet, some principles may be valid in most cases (i.e. in a certain percentage of observations), but not in all. They may not be called universal laws, but then do we have a term for such almost-always-valid principles? We must be careful here: what is usually called a "statistical law" concerns the probability of the event or the process involved, and not the confidence we have in the related principle or law (Carnap, 1974). This subjective feeling we have about universal laws is the main reason behind the inherent and definitive differences between the spirit, and thus in the nature, of $\mathrm{P}$ and those of $\mathrm{B}$. We may be tempted to imagine that because physical laws are universal, we can search for some universal explanation of physical processes or events. We may similarly tend to believe that because biological laws are not universal, we do not need to search for some universal explanation, but instead to study processes and events case by case. I intend to show in the following discussion that such differences in the spirit are illusory.

\section{Are $P$ and $B$ Different at All?}

Do universal laws exist in B? The process of natural selection or, more generally, the forces of evolution, appear to be a universal explanation of Life, as every living organism is subject to this "law". Indeed, although C. Darwin never called the principle he discovered a "law", the description would fit snugly into our previous definition of the term, as it drives every living entity. G. Mendel's genetic inheritance law also is a simple and generic principle of living entities, all of them carrying genetic information. Yet, these laws are not universal. The genetic inheritance law is a statistical law, because it expresses that sexual individuals depend on their parents' characteristics. Also, epigenetic and other Lamarckian-like processes are found to blur the clear view of a universal natural-selection law by adding to reproduction and evolution some processes that do not fit perfectly the variation-selection principle (Gaucherel \& Jensen, 2012; Por, 2006). Further, we admit that the processes and the laws we are talking about are the foundations of the observed events or entities, and cannot be deduced from some simpler processes or laws.

A careful scrutiny of the related facts tells us that both the biological laws mentioned show exceptions. Gene expressions sometimes depend on their environment (and not only on natu- 


\section{GAUCHEREL}

ral selection) when epigenetic processes occur, and may possibly be fixed across generations. Such exceptions to the initial biological laws seem very similar in nature to those of anomalies in the precession of the perihelion of Mercury first explained by A. Einstein in 1915 and later confirmed by Eddington's observations in 1919. These observations highlight the limits of the Newtonian law of gravitation, and encouraged some scientists to develop the general relativity theory. They demonstrate that B adopts similar ways of thinking to those that $\mathrm{P}$ does. Yet again, it seems intuitive that finding universal principles in $\mathrm{B}$ is harder and needs longer time than is the case in $\mathrm{P}$ (Keller, 2007).

Symmetrically, $\mathrm{P}$ is also experiencing studies on specific processes without seeking universality. Indeed, we saw that $P$ is using methods that are focusing on a specific process and a specific site when necessary. Geophysical processes, for example, when interpreting a specific tsunami or eruption, adopt very similar methods of study to those in a study of a specific cell division or protein production. However, these methods are hypothetico-deductive. Such examples are local and statistical, not universal. Yet, instead of biological examples, they may serve as bases on which to build inferences on more general principles. For example, the non-linear dynamic involved in the physical examples mentioned is a more generic principle that may be studied and developed for its possible universality.

Finally, these observations highlight the unclear definition we have of what is a law. They also illustrate the fact that a law is valid for a specific domain and at a specific date, and may be later replaced by a more accurate and more universal principle. As for theories explored by T. Kuhn, laws seem to carry for a while some paradigms that ultimately shift when the initial law is updated. Why should it be different in B from what it is in P? Natural selection, for example, might be a powerful law for a long time, then be modified and improved when we observe anomalies difficult to explain with its former version. This is exactly what Neo-Darwinism (with genetics and then epigenetic) brought to the pioneering work of Darwin.

\section{Redefinition of a Process}

In order to bring to light possible differences between $\mathrm{P}$ and $\mathrm{B}$ processes, or between $\mathrm{P}$ and $\mathrm{B}$ laws, we need today to revisit their insufficient definitions. Let's explore again what is a process and in which cases it progressively leads to a law. We have explained how a recurrent process starts defining a principle (or a law), and how a law without any exception defines a universal law. Neutral models are often of great help when they differentiate a process from a chance occurrence, i.e. when they detect any departures from neutrality.

Neutrality exhibits a wide range of facets. The first evidence of this fact appears in the observation that macroscale neutrality occurs with a purely neutral process, or arises as a limiting distribution by the aggregation of small-scale, non-random processes. In this case, non-neutral fluctuations tend to cancel each other out in the aggregate. S. A. Frank has clearly explained how neutral patterns describe patterns of nature that follow from simple constraints on information (Frank, 2009). Utilizing the powerful method of maximum entropy, he shows that each neutral generative model (Gaussian, exponential or geometric patterns) is a special case of a wider domain of the same neutral pattern. For example, any aggregation of processes that preserves information only about the mean and the variance tends to the Gaussian pattern.

Many patterns and processes in nature arise from limiting distributions. These neutral generative models form what are usually called probabilistic laws, for the reason that for a purely random process and a great number of events, the related distributions suffer almost no departure; the related laws suffer almost no exception. We should be careful here: these probability distributions are completely different from the natural laws previously discussed. Probabilistic laws concern chance (random processes); natural laws concern what is all but chance! In a sense, natural laws arise from neutral laws, as a clear departure from them: they are not random any more. Despite this difference, the basic concept behind a law, a pattern into which every process fits, is present in both cases.

Natural and probabilistic laws have another critical difference: only probabilistic laws exhibit a distance to the idealistic and pure (i.e. without departure) law. Testing a law indeed is rather a discrete task. In the case of a probabilistic law, the confidence level computed regarding to the known idealistic law quantifies this "distance" to it, i.e. how far from the pure (theoretical) law the observation is. In contrast, a natural law either exists or does not exist; it cannot be "in between". This definition of a law is appropriate for a universal law. But why should we not propose another definition inspired by probabilistic laws of what is a non-universal law? Why should we not interpret the rare failures of a universal law as a distance to the (supposed) universal law itself?

\section{Redefinition of a Law}

Suppose now we have correctly identified a non-random process at work. If such a process occurs recurrently under similar conditions, we may start to believe that an invariant principle is taking place here. We may observe some exceptions to this principle, say, with a random process occasionally taking the place of this non-random process. For example, the off-spring's gender is defined on the basis of their parents' genders, but this gender may occasionally change during the fetus' development, due to external factors. This is the case of reptile sex, when eggs are developing under various environmental temperatures. Finally, when the principle is systematic, we call it a law. With such a definition, Mendel's genetic inheritance law is no more a universal law, because it does not take into account environmental factors that can perturb it, because it is "almost" always true for sexual reproduction.

When we have in mind a biological law, such as the so-called Mendel's law, we do not think about a universal law, but rather a law against which some processes might be found. This example highlights a critical difference between variations found in processes and variations found in laws. Carnap is a pioneer philosopher having insisted on this difference, although not in modern terms (Carnap, 1974). A process is always observed to possess some stochasticity, either because of inherent stochasticity, or because of sampling stochasticity. In other words, a noise process is detectable in so far as it is possible to reduce enough noise around it. But the process is always present. This analysis is related to probabilistic laws, as the experimenter has to manage the chance superimposed on the present process. In other cases, the process might be present or not, depending on the various internal and external factors of the entity involved. The consequent analysis is here related to the universality of the law, rather than to the chance element present in the process. 
Therefore, the associated law is universal or not, according as the process has a systematic presence or not. However, when the process is systematically present except in one isolated case, could we not say that it is "almost" universal?

Two types of failure of a process can be found in nature. A non-random process might be more or less visible, emerging from a noisy background, and leading to the illusion that the principle is not a strong principle. Also, such a process is occasionally replaced by, and not just hidden by, a random process (or another process). It may have a certain probability to occur and to be observed. I propose here that it is possible to interpret the latter type of failure with a probabilistic view similar to the former type of failure, but at the law level instead of at the process level. Laws are universal when they suffer absolutely no exception, but none of them are truly universal. Most of them are only rarely violated by the absence of their related process, and the replacement of it by another process. Hence, they have a probability of validity of less than unity. I suggest that every law possesses a probabilistic nature, with a probability to be valid that could be defined as equal to unity in the case of universal laws.

Carnap (1974) uses the notion of logical probability (also called inductive probability), which he borrowed from John Maynard Keynes, to handle the statement that a law could be probabilistic instead of universal. Carnap asks: "How well established is the law?" He then provides a possible answer: "This hypothesis is confirmed to degree .8 on the basis of the available evidence", which thus "expresses a logical relation between a sentence that states the evidence and a sentence that states the hypothesis". This form of probability is quite different from the previously mentioned statistical probabilities used to control the stochasticity of a process. With logical probability, we are no longer talking about its related process, but rather about its law and the confidence (called "degree of confirmation" by Carnap) we have in it. Such logical probability is "especially useful in meta-scientific statements" and could, similarly to usual probabilities, be interpreted by a frequency meaning. Logical probabilities offer the opportunity to make (logical and) quantitative judgments on laws, on the basis of experience.

\section{Differences between $P$ and $B$ Laws: Number of Events}

We often have the feeling that we are less confident with respect to biological laws than we are to physical laws. This would have been so if biological laws were more uncertain than physical ones. Following the previous definition of a law, all goes as if biological laws had lower logical probabilities than physical ones. In a sense, this logical probability quantifies how far removed a particular law is from the universal law it could be associated with. Whatever the probability of a stochastic process, we may be more or less confident about the law with which it is associated.

I propose here the thesis that physical and biological disciplines exhibit different logical probabilities. Moreover, I will try to explain why I think physical and biological laws have differing logical probabilities. We did not find any difference in nature between $\mathrm{B}$ and $\mathrm{P}$, although we collected in the previous sections several (dependent) clues that sometimes differ among themselves, namely scale, irreversibility, or number of events.

The number of events, or of interactions of entity components, is a fruitful property to explore in this context. P usually handles a larger number of events or of components than B, as living entities are built on bricks of inert entities, and not the other way around. To illustrate this assertion, just think about the Avogadro number, which is defined as the ratio of the number of constitutive atoms (or molecules) in a sample to the amount of any substance (a mole). The Avogadro constant $(6.02 \times 1023)$ is a kind of scaling factor between macroscopic and microscopic scales, or, more precisely, between the scale of an entity and the scale of its components. The assertion on the number of components is not about the scale per se of a particular $\mathrm{P}$ or $\mathrm{B}$ process, but intends instead to point attention to the ratio of the scales. Conceivably, this scaling ratio is not always higher in $\mathrm{P}$ than in $\mathrm{B}$. We saw counter-examples at the beginning of this text, which might even be another reason that blurs the frontiers between $\mathrm{P}$ and $\mathrm{B}$. At least, this is a difference that common sense can accept: events and constitutive entities in these two disciplines differ in number in general.

So, P usually appears to have highly stable and reproducible processes, often due to the higher number of components present in it. This partly explains why physical observations converge to neutral statistical laws, due to the "law of large numbers” (Frank, 2009). This law states that the average of the results obtained from a large number of trials should be close to their expected value, and will tend to become closer as more trials are performed. Statistical laws appear to be limiting distributions reached with confidence if a sufficiently large number of entities interact to produce the observed process. With lower numbers of components, it is more difficult and less frequent to observe with confidence the limiting distribution.

Similarly, I hypothesize that this number of events is directly related to the fact that natural laws are either valid or invalid, and more or less certain (i.e. valid or invalid), depending on the discipline being considered. We could say too: With lower numbers of events, we are more likely to observe departures from the universal law, i.e. a case where the process fails to occur. I suggest labeling as a robust law a probabilistic law which is close to its associated universal law. Hence, a robust law has a logical probability close to unity. With such a definition, we can say that B's laws are less robust than P's laws, as they are pushed away from their universal laws by the relatively low number of events and entities they involve.

\section{Discussion and Synthesis}

It has been illustrated that $\mathrm{P}$ often searches for universal laws to explain physical events, while B usually focuses on specific processes, without a search for universality. Of course, this dichotomy is probably less sharp than it appears at first sight. Some authors explain how these methods may be merged into a common, more powerful and more complete scientific method (Harte, 2002). Some others insist on the fact that these P and B cultures are and should remain separated (Keller, 2007). A natural question follows: Are the $\mathrm{P}$ and $\mathrm{B}$ cultures so different?

The feeling that $\mathrm{P}$ and B represent a "clash of two cultures" is, in my opinion, partly related to the other feeling that inert entities and living entities are different. This point has never been demonstrated up to now, as we do not have today a clear definition of what is Life and how Life appeared (Schrödinger, 1945). In agreement with other writers (Pombo et al., 2012), I have tried to explain with examples why unicity, contingency and irreversibility, the three properties sometimes proposed to differentiate living entities from inert entities, are not relevant 


\section{GAUCHEREL}

or, at least, not sufficient in themselves to split the scientific culture into $\mathrm{P}$ and $\mathrm{B}$ cultures.

This feeling about $\mathrm{P}$ and B cultures probably also comes from the weak knowledge we have of what our scientist neighbor is doing, as only very few physicists and biologists shift to (and stay in) each other's disciplines. This remark does not mean that physicists and biologists are not collaborating, but that to switch disciplines involves each recognizing the other's entity and event specificities, the other's ontology. It may also involve adopting another scientific method, although, as I have also explained, the rise of NM in both B and P should illustrate a shared approach the two domains practice in their respective research work.

With a persistent feeling that $\mathrm{B}$ and $\mathrm{P}$ may indeed be different in nature, I have tried to explore the complex role of the concepts of scale and number of events in the two domains to interpret possible intrinsic differences. It appeared that it is always possible to identify processes and entities involving similar scales and numbers of entities between B and P. Yet, in general, physical entities (and events) involve a higher number of entities than do biological entities. In $\mathrm{P}$, this greater number of events usually leads physical processes to more easily converge towards the ideal limiting distribution. $P$, with its highly stable and reproducible events, therefore justifies a higher level of confidence with regard to the processes observed.

The multiplicity of the components in a physical system tends to average its behavior more than would be the case in B. Following Carnap, I am proposing here the first thesis that a law might occasionally be violated, rather than saying that the law is wrong and should be replaced. Such occasional violation could be interpreted as a logical probability of the law, based on a meta-scientific judgment. Whatever the probability of the process being studied, we may still be more or less confident about the law it is associated with.

The variations inherent in biological entities suggest recourse to small samples with high heterogeneity among them. This is tantamount to implying low confidence in the laws. Based on the new definition of a law that we have referred to, I propose here the second thesis that physical and biological disciplines exhibit different "logical probabilities". We therefore have differing degrees of confidence in their laws. Another way of expressing this view would be to say that it is as if living entities would require "relaxed" confidence levels for their laws compared to physical laws. Say, for example, that we only have a $99 \%$ chance that Natural Selection would apply to Life, whereas the second law of thermodynamics has a $99.9 \%$ chance to apply to a system. In some cases further away, found on Earth or on the planet Pandora for example, we may find living entities based on other evolution rules (Gaucherel \& Jensen, 2012). Could it be that biological laws are less robust than physical laws?

This proposition does not suggest that all physical laws are robust. We have seen non-universal laws in $\mathrm{P}$ that are nevertheless relatively robust. Let's go back to the two previously mentioned laws in P: the second law of thermodynamics and the law of universal gravitation. The "fluctuations theorem" in thermodynamics states that out-of-equilibrium systems behave similarly to small fluctuations of systems at equilibrium (Evans et al., 1993), with a non-zero statistical probability to reduce the system entropy. Therefore, such situations momentarily violate the second law of thermodynamics, which states that systems never decrease their entropy. This violation is due to random fluctuations in the process itself, reminding us that the second law is a statistical law.

On the other hand, Newton's law of gravitation has been invalidated for strong gravitational fields, such as the ones encountered in the famous 1919 Eddington experiment on the anomalous precession of the perihelion of Mercury. Thanks to that experiment, the domain of the validity of the Newtonian law of gravity has been redefined, thus losing its "universal" attribute. It is not that the process behind gravitation was not clearly measured (due to chance or noise), but rather that the process itself was no more the same between weak and strong gravitational fields. We are here in the presence of a probabilistic law, as it remains valid in a relative proportion of cases. The Newtonian law of gravity is relatively robust, and is today less robust than the general theory of relativity.

In these physical examples, old and strict laws (i.e. suffering no exception) were modified into new statistical or probabilistic laws (with rare but acceptable exceptions). Yet these exceptions have completely different origins: they arise in the processes associated with the older statistical laws, and in the confidence we have in the later probabilistic laws.

If this concept turns out to be true, laws may be found both in $\mathrm{P}$ and $\mathrm{B}$ disciplines, but with a kind of "conceptual continuum” between very robust laws in $\mathrm{P}$ (i.e. with a very low probability of being refuted by a single event or entity) and less robust laws in B (with a probability of temporary or local exceptions being found).

To extrapolate this definition, I would like to suggest that laws in economics (or probably in most human-related sciences) are even less robust than they are in B. The numbers of humans or of events in human society are lower than the numbers involved in $\mathrm{B}$, and this may also explain why their associated laws show so little robustness. Conversely, we may extrapolate this definition into other directions of the robustness. Mathematics, daily handling infinite quantities of data, could have the most robust laws, despite the fact that it is not an empirical discipline. Hence, differences between physical and biological laws could be quantitative (linked to the laws' probabilities) instead of qualitative (either universal or not), as common sense would first suggest.

\section{Conclusion}

P and B do not present a sharp "epistemic rupture” (Keller, 2007), but rather a conceptual continuum of scientific disciplines. $\mathrm{P}$ and $\mathrm{B}$ both search for some universal laws, but these laws may exhibit different degrees of robustness relative to the probability that they encounter an exception. The laws' robustness could be measured on the basis of logical probabilities. If this concept is revealed to be true, laws may be found both in B and in $\mathrm{P}$ disciplines, but with a continuum between very robust laws (often found in $\mathrm{P}$, with a very low probability of refutation by an observed event) and less robust laws in B (with a nonnull probability of occasional violation or refutation by locally found exceptions).

So, why not search for universal laws in the life sciences, bearing in mind that they may occasionally be violated, although with a low probability? In a second stage, we would try to minimize our probabilistic view of the living entities studied, by increasing our confidence in their associated laws (i.e. by reducing the uncertainty we have regarded the laws and the explanation of observations). It is possible that we may not be 


\section{GAUCHEREL}

able to increase our confidence in B's laws. This confidence is sometimes linked to the relatively low number of entities and/or interactions involved. In this case, a universal law, playing the role of a theoretical and limiting law, may never be reached, and we would need to radically change the law studied, as we have usually done up to now. To sum up, $\mathrm{P}$ and $\mathrm{B}$ do not differ; rather they represent the two poles of a unique scientific culture. This scientific culture may just be modulated by a probabilistic understanding of the world.

\section{Acknowledgements}

I would like to express my warm thanks to P. Huneman and F. Munoz for their patient reading of the earlier versions of this paper, and Allan Bailur for English editing.

\section{REFERENCES}

Balian, R. (1991). From microphysics to macrophysics: Methods and applications of statistical physics. Berlin: Springer Verlag.

Barabasi, A. L., \& Albert, R. (1999). Emergence of scaling in random networks. Science in Context, 286, 509-512

Beaulieu, J.-P., Bennett, D. P., Fouqué, P., Williams, A., Dominik, M. et al. (2006). Discovery of a cool planet of 5.5 Earth masses through gravitational microlensing. Nature, 439, 437-440. doi:10.1038/nature04441

Benhamou, S. (2007). How many animals really do the Levy walk? Ecology, 88, 1962-1969. doi:10.1890/06-1769.1

Carnap, R. (1974). An introduction to the philosophy of science. Mineola: Dover science book.

Cartwright, N. (1983). How the laws of physics lie. Oxford: Oxford University Press.

Chase, J. M. (2005). Towards a really unified theory for metacommunities. Functional Ecology, 19, 182-186.

D’Souza, R. M., Borgs, C., Chayes, J. T., Berger, N., \& Kleinberg, R. D. (2007). Emergence of tempered preferential attachment from optimization. Proceedings of the National Academy of Sciences of the United States of America, 104, 6112-6117. doi:10.1073/pnas.0606779104

Evans, D. J., Cohen, E. G. D., \& Morriss, G. P. (1993). Probability of second law violations in shearing steady states. Physical Review Letters, 71, 2401-2404. doi:10.1103/PhysRevLett.71.2401

Fisher, R. A. (1966). The design of experiments (8th ed.). Edinburgh: Hafner.

Frank, S. A. (2009). The common patterns of nature. Journal of Evolutionnary Biology, 22, 1563-1585. doi:10.1111/j.1420-9101.2009.01775.x

Gardner, R. H., Milne, B. T., Turner, M. G., \& O’Neill, R. V. (1987). Neutral models for the analysis of broad-scale pattern. Landscape Ecology, 1, 19-28. doi:10.1007/BF02275262

Gaucherel, C. (2011). Self-organization of patchy landscapes: Hidden optimization of ecological processes. Ecosystem \& Ecography, 1.

Gaucherel, C., Fleury, D., Auclair, A., \& Dreyfus, P. (2006). Neutral models for patchy landscapes. Ecological Modelling, 197, 159-170. doi:10.1016/j.ecolmodel.2006.02.044

Gaucherel, C., \& Jensen, H. J. (2012). Origins of evolution: Non acquired characters dominates over acquired characters in changing environment. Journal of Theoretical Biology, in Press. doi:10.1016/j.jtbi.2012.02.028

Gould, S. J. (1989). Wonderful life: The burgess shale and the nature of history. New York.

Halley, J. M., Hartley, S., Kallimanis, A. S., Kunin, W. E., Lennon, J. J., \& Sgardelis, S. P. (2004). Uses and abuses of fractal methodology in ecology. Ecology Letters, 7, 254-271. doi:10.1111/j.1461-0248.2004.00568.x

Harte, J. (2002). Toward a synthesis of the Newtonian and Darwinian worldviews. Physics Today, 29-34. doi:10.1063/1.1522164

Hubbell, S. (2001). The unified neutral theory of biodiversity and biogeography. Princeton, NJ: Princeton University Press.

Kauffman, S. (1993). Origins of order: Self-organization and selection in evolution. Technical monograph. Oxford: Oxford University Press.

Keller, E. F. (2007). A clash of two cultures. Nature, 445, 603-603

Kimura, M. (1983). The neutral theory of molecular evolution. Cambridge: Cambridge University Press.

doi:10.1017/CBO9780511623486

Lovelock, J. (2003). The living Earth. Nature, 426, 769-770. doi:10.1038/426769a

Mayr, E. (2004). What makes biology unique? Cambridge: Cambridge University Press. doi:10.1017/CBO9780511617188

Morris, S. C. (2010). Evolution: Like any other science it is predictable. Philosophical Transactions of the Royal Society B, 365, 133-145. doi:10.1098/rstb.2009.0154

Nicolis, G., \& Prigogine, I. (1977). Self organization in nonequilibrium systems. New York.

Nitecki, M. H., \& Hoffman, A. (1987). Neutral models in biology. Oxford: Oxford University Press.

Pesic, P. (2002). Seeing double: Shared identities in physics, philosophy, and literature. Cambridge: The MIT Press,

Pombo, O., Torres, J. M., Symons, J., \& Rahman, S. (2012). Special sciences and the unity of science, vol. 24. Logic, Epistemology, and the Unity of Science.

Por, F. D. (2006). The actuality of Lamarck: Towards the bicentenary of his Philosophie Zoologique. Integrative Zoology, 1, 48-52.

Scanlon, T. M., Caylor, K. K., Levin, S. A., \& Rodriguez-Iturbe, I. (2007). Positive feedbacks promote power-law clustering of Kalahari vegetation. Nature, 449, U209-U204. doi:10.1038/nature06060

Schrödinger, E. (1945). What is life? The physical aspect of the living cell and mind and matter. Cambridge: Cambridge University Press.

Tegmark, M. (2003). Parallel universes. Scientific American, 40-51. doi:10.1038/scientificamerican0503-40

Trepl, L., \& Voigt, A. (2011). The classical holism-reductionism debate in ecology. In A. Schwarz, \& K. Jax (Eds.), Ecology revisited. Reflecting on concepts, advancing science (pp. 45-83). Berlin: Springer. doi:10.1007/978-90-481-9744-6

Viswanathan, G. M., Buldyrev, S. V., Havlin, S., da Luz, M. G. E., Raposo, E. P., \& Stanley, H. E. (1999). Optimizing the success of random searches. Nature, 401, 911-914. doi:10.1038/44831 